\title{
Promoter Mutation Analysis of LEPROTL1 Gene in Acute Leukemias and Solid Tumors
}

\author{
Ha Yoon Mo ${ }^{a}$ Min Sung Kim ${ }^{a} \quad$ Nack Gyun Chung ${ }^{b}$ Nam Jin Yoo ${ }^{a}$ Sug Hyung Lee \\ a Department of Pathology, College of Medicine, The Catholic University of Korea, Seoul, South Korea; \\ ${ }^{b}$ Department of Pediatrics, College of Medicine, The Catholic University of Korea, Seoul, South Korea
}

Dear Editor,

The leptin receptor overlapping transcript-like 1 gene (LEPROTL1) encodes a membrane protein $[1,2]$. To date, the only known function of this gene product is to suppress growth hormone activity in the liver [2]. The cancer-related functions of LEPROTL1 remain unknown. According to the Human Protein Atlas database (www.proteinatlas.org), LEPROTL1 is widely expressed in human tissues, but its expression in most cancer types is negative, suggesting that loss of LEPROTL1 could be related to tumorigenesis. A recent study analyzed genome-wide somatic mutations in promoters in breast cancers and identified promoter mutations in several genes [3], including LEPROTL1, which resulted in its decreased expression, suggesting a loss of function [3]. Interestingly, the promoter mutations repeatedly occur at 2 hotspots (chr8:29952919 and chr8: 29952921). Alterations in promoter regions such as somatic mutation and aberrant methylation are known to play a role in cancer pathogenesis [4]. Together, these data suggest that promoter mutation in the LEPROTL1 gene might be prevalent and play a role in the development of breast cancer as well as other cancers.

For this study, we analyzed somatic promoter mutations of the LEPROTL1 gene using genomic DNA from bone marrow aspirates of 1,011 hematologic tumors (acute myelogenous leukemia [AML], acute lymphoblastic leukemia [ALL], mul- tiple myeloma, and myelodysplastic syndrome) (Table 1) by polymerase chain reaction (PCR) and single-strand conformation polymorphism (SSCP) assay. We also analyzed the DNA from 127 non-Hodgkin lymphomas (NHL) and 1,545 epithelial and mesenchymal tumors of various origin (Table 1). For the solid tumors, malignant and normal cells were selectively procured by microdissection [5]. Approval for this study was obtained from the Institutional Review Board of the Catholic University of Korea's College of Medicine.

Because LEPROTL1 promoter mutations have been focused in a narrow region (chr8:29952919 and chr8:29952921) [3], we amplified this region by PCR with the following primers: forward: 5-ACACCCGCGGAGAAGAGAG-3, reverse: 5-GGAGACCCAAGACGCGCTAC-3, and subsequently analyzed the PCR products by SSCP. Genomic DNA from tumor cells and normal cells (remission bone marrow cells from leukemia cases) was used in this study. The PCR-SSCP method has previously been described [5]. After SSCP, direct DNA sequencing reactions were performed in the cancers with mobility shifts.

PCR and subsequent SSCP analysis detected somatic promoter mutations of LEPROTL1 in 3 cancers (1 case of childhood ALL, 1 laryngeal carcinoma, and 1 angiosarcoma). The matched normal samples were also sequenced but did not show the mutations. All of these mutation sites de- tected in our study (Table 1) overlap with the hotspots identified in the earlier study [3]. The ALL patient (a 6-year-old girl) with the LEPROTL1 mutation was diagnosed as having B lymphoblastic leukemia with $\mathrm{t}(12 ; 21)(\mathrm{p} 13 ; \mathrm{q} 22)$;TEL-AML1 (ETV6RUNX1). Cytogenetic analysis of the bone marrow demonstrated the following karyotype in detail: 46,XX,del(11)(q14q21),t(11; $18)(\mathrm{p} 15 ; \mathrm{q} 12)$, add (12)(p11.2)[3] / 46,XX[17].ish $\mathrm{t}(12 ; 21)(\mathrm{p} 13 ; \mathrm{q} 22)(\mathrm{ETV} 6+$, RUNX1+;RUNX1 + 1,ETV6 + 1)[20]. After chemotherapy, she achieved complete remission and has been healthy for 2.5 years without relapse. While the specificity of SSCP used in this study was addressed by the recurrent sequencing of positives with the earlier data, its sensitivity was not. It is possible that the negative mutation status could be a result of the failure of SSCP to detect a specific variant. Under suitable conditions, SSCP is capable of detecting $>90 \%$ of mutations occurring within any sequence, and the sensitivity of PCR-SSCP is generally believed to be high if the fragments are $<200 \mathrm{bp}$ [6]. Because we analyzed the samples with products $<200$ bp by SSCP, it is unlikely that we would miss the LEPROTL1 mutations.

One goal of this study was to identify whether the promoter mutation found in

Ha Yoon Mo and Min Sung Kim contributed equally to this work.

\section{KARGER}

(c) 2019 S. Karger AG, Basel
Sug Hyung Lee, MD

Department of Pathology, College of Medicine

The Catholic University of Korea

505 Banpo-dong, Socho-gu, Seoul 137-701 (South Korea)

www.karger.com/aha 
Table 1. LEPROTL1 promoter mutation in tumors from 2,683 patients

\begin{tabular}{|c|c|c|c|c|}
\hline \multirow[t]{2}{*}{ Type of tumor } & \multirow{2}{*}{$\begin{array}{l}\text { Tumors, } \\
n\end{array}$} & \multicolumn{2}{|c|}{ LEPROTL1 promoter } & \multirow[t]{2}{*}{ Location } \\
\hline & & wild-type & mutation (\%) & \\
\hline Adulthood AML & 299 & 299 & $0(0)$ & \\
\hline Adulthood ALL & 176 & 176 & $0(0)$ & \\
\hline Childhood AML & 31 & 31 & $0(0)$ & \\
\hline Childhood ALL & 361 & 360 & $1(0.5)$ & chr8:29952919G >C \\
\hline Multiple myeloma & 76 & 76 & $0(0)$ & \\
\hline Myelodysplasia & 68 & 68 & $0(0)$ & \\
\hline Non-Hodgkin lymphoma & 127 & 127 & $0(0)$ & \\
\hline Non-small-cell lung cancer & 170 & 170 & $0(0)$ & \\
\hline Gastric carcinoma & 247 & 247 & $0(0)$ & \\
\hline Colorectal carcinoma & 438 & 438 & $0(0)$ & \\
\hline Prostate carcinoma & 270 & 270 & $0(0)$ & \\
\hline Ovarian epithelial tumors & 25 & 25 & $0(0)$ & \\
\hline Ovarian stromal tumors & 115 & 115 & $0(0)$ & \\
\hline Hepatocellular carcinomas & 43 & 43 & $0(0)$ & \\
\hline Hepatoblastomas & 13 & 13 & $0(0)$ & \\
\hline Esophageal squamous-cell carcinomas & 32 & 32 & $0(0)$ & \\
\hline Laryngeal squamous-cell carcinomas & 19 & 18 & $1(5.3)$ & chr8:29952921C>T \\
\hline Leiomyoma & 68 & 68 & $0(0)$ & \\
\hline Gastrointestinal stromal tumors & 21 & 21 & $0(0)$ & \\
\hline Sarcomas & 56 & 55 & $1(1.8)$ & chr8:29952921C>G \\
\hline Meningioma & 28 & 28 & $0(0)$ & \\
\hline Total & 2,683 & 2,680 & $3(0.11)$ & \\
\hline
\end{tabular}

AML, acute myelogenous leukemia; ALL, acute lymphoblastic leukemia.

breast cancer is common to other cancer types, including hematologic malignancies. The mutation prevalence in ALL $(0.5 \%)$, laryngeal carcinoma $(5.3 \%)$, and sarcoma $(1.8 \%)$ is not significantly different $(p>0.05)$ from that in breast cancers (1.7\%) [3]. Because LEPROTL1 is ubiquitously expressed in tissues including breast tissue, and is lost in almost all types of cancers, we expected to find some mutations in the large cohort of hematologic and solid tumors. However, we detected the promoter mutation in only 3 cancers, which is a very low prevalence $(3 / 2,683[0.11 \%])$. In

\section{References}

1 Huang $\mathrm{Y}$, Ying $\mathrm{K}$, Xie Y, Zhou Z, Wang W, Tang R, et al. Cloning and characterization of a novel human leptin receptor overlapping transcript-like 1 gene (LEPROTL1). Biochim Biophys Acta. 2001 Jan;1517(2):327-31.

2 Touvier T, Conte-Auriol F, Briand O, Cudejko C, Paumelle R, Caron S, et al. LEPROT and LEPROTL1 cooperatively decrease hepatic growth hormone action in mice. J Clin Invest. 2009 Dec;119(12):3830-8. the hematologic tumors, only 1 ALL case exhibited the mutation.

These data indicate that LEPROTL1 promoter mutations may be rare in both common hematologic and solid cancers. The discovery of the recurrent LEPROTL1 promoter mutations in breast cancers offered an opportunity for developing therapeutic and diagnostic tools targeting the mutations. Our results, however, indicate that applications targeting the LEPROTL1 mutations may not be clinically available in acute leukemia in the future due to the low incidence of the LEPROTL1 mutation.
3 Rheinbay E, Parasuraman P, Grimsby J, Tiao G, Engreitz JM, Kim J, et al. Recurrent and functional regulatory mutations in breast cancer. Nature. 2017 Jul;547(7661):55-60.

4 Je EM, Kim MR, Min KO, Yoo NJ, Lee SH. Mutational analysis of MED12 exon 2 in uterine leiomyoma and other common tumors. Int J Cancer. 2012 Sep;131(6):E1044-7.

\section{Acknowledgements}

This study was supported by grants from National Research Foundation of Korea (2017R1D1A1B03027840).

\section{Disclosure Statement}

The authors report no conflicts of interest.

5 Heng J, Guo X, Wu W, Wang Y, Li G, Chen $\mathrm{M}$, et al. Integrated analysis of promoter mutation, methylation and expression of AKT1 gene in Chinese breast cancer patients. PLoS One. 2017 Mar;12(3):e0174022.

6 Hayashi K. PCR-SSCP: a simple and sensitive method for detection of mutations in the genomic DNA. PCR Methods Appl. 1991 Aug; 1(1):34-8. 